\title{
LEVEL SET METHODS FOR DYNAMIC TOMOGRAPHY
}

\author{
Yonggang Shi \\ William Clem Karl \\ Information Systems and Sciences Laboratory \\ Electrical and Computer Engineering Department \\ Boston University \\ Email: \{yshi,wckarl $\} @$ bu.edu
}

\begin{abstract}
In this paper, we propose a novel variational framework for the reconstruction of dynamic objects from sparse and noisy tomographic data. Using an object-based scene model, we developed a general object dynamic model based on a one to one and differentiable mapping. We then propose a novel distance between curves to incorporate the object dynamics into the variational framework. For the minimization of the energy function, we developed a coordinate descent algorithm based on the level set methods. Experimental results for reconstructing a sequence of multiple dynamic objects are presented.
\end{abstract}

\section{INTRODUCTION}

The goal of dynamic tomography is to provide functional information of objects from the tomographic data observed over time. Its applications include cardiac computed tomography, dynamic studies in nuclear medicine such as myocardial perfusion imaging, blood pool imaging and renal imaging, etc. The challenges of dynamic tomography generally include: sparsity of projection data, high computational and memory requirement for this 3-D or 4-D problem, ill-posedness of the inverse problem, and the modeling of dynamics. Among these factors, the most challenging is the modeling of motion and its incorporation into the reconstruction process. Good temporal models will not only incorporate prior knowledge about scene dynamics, they will also reduce the dimension of unknowns and thus reduce the computational cost, and improve the robustness of the algorithm to noise.

In the field of nuclear medicine, considerable research has been done to model the dynamics of the image sequence at the pixel level. By using compartmental models from biological research, parametric models can be introduced to model the dynamic process at each pixel [1]. To reconstruct

This work was partially supported by The Air Force Office of Scientific Research under Grant F49620-03-1-0257, The National Institutes of Health under Grant NINDS 1 R01 NS34189, and The Engineering research centers program of the NSF under award EEC-9986821 the image sequence, a set of parameters then need to be estimated for every pixel. Along this line, various algorithms have been developed [2,3]. However, these methods still results in high dimensional challenging optimization problems, and object-level motions are also hard to taken into account with these methods.

In many challenging tomography problems, the focus is on the extraction of information about a set of objects in the underlying scene. Direct estimation of parametric object models from projection data were first proposed in [4]. Recently, methods based on curve evolution and level set methods [5] become very popular for solving various static inverse problems [6,7]. To this end, parametric object models have also been applied to reconstruct a single dynamic objects, for example $[8,9]$. But these methods were not able to incorporate object motion models into the reconstruction.

In this paper, we propose a novel variational method for the reconstruction of dynamic objects directly from challenging tomographic data. We model the motion of objects explicitly and propose a novel shape distance to incorporate our object dynamic model into the reconstruction process. Our methods can reconstruct dynamic objects from extremely sparse projection data. Using efficient level set methods for object boundary representation, we can also reconstruct multiple dynamic objects simultaneously.

The rest of the paper is as follows. In section 2, we present our model of object dynamics. The variational framework is proposed in Section 3. Experimental results are presented in Section 4. Finally, conclusions are made in Section 5.

\section{OBJECT-BASED MODELING}

Let $f(\mathbf{x}, k)(k=1,2, \cdots, \mathbf{K})$ denote an image sequence consisting of $\mathbf{K}$ scenes and each scene is defined over a domain $\mathbf{D} \subset \mathbf{R}^{\mathbf{2}}$. We assume the scene at time $k$ is composed of $N$ regions: $\left[\Omega_{k}^{1}, \cdots, \Omega_{k}^{N}\right]$. The boundaries of these $N$ regions can be represented by $L(L \leq N)$ curves $\left[C_{k}^{1}, \cdots, C_{k}^{L}\right]$. Since these curves can intersect, the number of regions they delineate is larger than the number of curves. The inten- 
sity of each region is assumed uniform and denoted as $\beta_{k}^{n}$. We stack the intensity of all regions into a vector $\beta_{k}=$ $\left[\beta_{k}^{1}, \cdots, \beta_{k}^{N}\right]$.

\subsection{Dynamic model}

Based on our image sequence model, we model the dynamics of the image sequence as composed of two parts: the dynamics of the object boundaries and the dynamics of the intensities.

For the dynamics of the object boundaries at time $k$, we model it as composed of two parts. A one to one and differentiable mapping $A_{k}: \mathbf{R}^{2} \rightarrow \mathbf{R}^{2}$ is introduced to account for the part of the dynamics that is known a priori and another mapping $W_{k}: \mathbf{R}^{\mathbf{2}} \rightarrow \mathbf{R}^{\mathbf{2}}$ is used to represent unknown and probably irreversible small perturbations to the boundary. Using these two transformations, we express the dynamics of the object boundary as follows:

$$
C_{k+1}^{l}=A_{k}\left(C_{k}^{l}\right)+W_{k}, \quad l=1, \cdots, L
$$

i.e., for any $\mathrm{x} \in C_{k}^{l}$, there is a point $\mathrm{x}^{\prime} \in C_{k+1}^{l}$ such that $\mathbf{x}^{\prime}=A_{k}(\mathbf{x})+W_{k}(\mathbf{x})$.

For intensity dynamics, we use a simple auto-regressive model:

$$
\beta_{k+1}=B_{k} \beta_{k}+u_{k}
$$

where $B_{k}$ is the system matrix and $u_{k}$ is a random vector of Gaussian distribution with zero mean and covariance matrix $P_{k}$.

\subsection{Observation model}

Let $Y_{k}^{i}(i=1, \cdots, M)$ denote the $i$-th component of the clean projection data at time $k$ and $H_{k}^{i}(\cdot)$ the projection kernel. We can express the observation model as follows:

$$
Y_{k}^{i}=\sum_{n=1}^{N} \beta_{k}^{n} \int_{\Omega_{k}^{n}} H_{k}^{i}(\mathbf{x}) d \mathbf{x}
$$

We can also express this observation model in a vector form:

$$
Y_{k}=H_{k} \beta_{k}
$$

where $H_{k}$ is an $M \times N$ matrix with its elements defined as: $H_{k}(i, n)=\int_{\Omega_{k}^{n}} H_{k}^{i}(\mathbf{x}) d \mathbf{x}$. Note that $H_{k}$ is itself a function of $C_{k}^{l}(l=1, \cdots, L)$, so our dynamic tomography observation model is indeed nonlinear with respect to the unknowns.

In applications, noisy versions of the clean data are usually observed. Let $y_{k}$ denote the noisy observation at time $k$. We assume a general noise model and express the conditional distribution of $y_{k}$ given $Y_{k}$ as:

$$
y_{k} \sim \mathbf{p}\left(y_{k} \mid Y_{k}\right) \quad k=1,2, \cdots, K
$$

\section{A VARIATIONAL FRAMEWORK}

To reconstruct the image sequence directly from the sparse and noisy projection data, we propose a variational framework. With this approach, all the information and regularization terms can be combined into a single energy function. Tractable optimization algorithms can also be developed to estimate model parameters.

In our variational framework, an energy function $E$ is defined as a weighted sum of four terms:

$$
E=E_{d}+\lambda_{s} E_{s}+\lambda_{t} E_{t}+\lambda_{i} E_{i},
$$

where $E_{d}$ is the data fidelity term, $E_{s}$ is the spatial boundary smoothness term, $E_{t}$ is the temporal boundary smoothness term, and $E_{i}$ is the temporal intensity smoothness term. The details of their definition will be presented in the follows. $\lambda_{s}, \lambda_{t}$, and $\lambda_{i}$ are non-negative regularization parameters used to weight these different terms. With the energy well defined, we formulate our reconstruction problem as an energy minimization problem:

$$
(\mathbf{C}, \beta)=\arg \min _{(\mathbf{C}, \beta)} E(\mathbf{C}, \beta)
$$

where $\mathbf{C}=\left[C_{1}^{1}, \cdots, \mathbf{C}_{K}^{L}\right]$ and $\beta=\left[\beta_{1}^{1}, \cdots, \beta_{K}^{N}\right]$.

For the data fidelity term, we define $E_{d}$ to be proportional to the negative log-likelihood of the data, i.e.:

$$
E_{d}=-\log \mathbf{p}\left(\mathbf{y}_{k} \mid Y_{k}\right)
$$

Common choices of $\mathbf{p}$ are Gaussian and Poisson distributions.

The spatial boundary smoothness term $E_{s}$ is defined as the sum of the length of all the boundaries:

$$
E_{s}=\sum_{k, l} \int_{C_{k}^{l}} d C_{k}^{l}
$$

By incorporating $E_{s}$ as a regularization term, smoothness of the boundaries can be enforced [10].

The temporal boundary smoothness term $E_{t}$ is designed to incorporate the boundary dynamics model in Eq.(1). This is a challenging task because $W_{k}$ is unknown and correspondences between points on the two curves $C_{k+1}^{l}$ and $A_{k}\left(C_{k}^{l}\right)$ are hard to establish in reality. Motivated by the $L^{p}$ distance between vectors, we propose a novel distance between curves and penalize the distance between $C_{k+1}^{l}$ and $C_{k}^{l}$ to incorporate the boundary dynamics.

Let $C_{a}$ and $C_{b}$ be two closed curves in $\mathbf{R}^{2}$. Their difference region is $\pi\left(C_{a}, C_{b}\right)$ which are the set of points inside $C_{a}$ but outside $C_{b}$ or inside $C_{b}$ but outside $C_{a}$. We define the distance of these two curves as:

$$
d_{p}\left(C_{a}, C_{b}\right)=\left(\int_{\pi\left(C_{a}, C_{b}\right)}\left(d_{C_{a}}(\mathbf{x})+d_{C_{b}}(\mathbf{x})\right)^{p-1} d \mathbf{x}\right)^{1 / p}
$$


where $p>0, d_{C_{a}}(\mathbf{x})$ and $d_{C_{b}}(\mathbf{x})$ are the distance functions of $C_{a}$ and $C_{b}$, respectively. Using our distance measure, the temporal boundary smoothness term $E_{t}$ is:

$$
E_{t}=\sum_{k, l}\left(\int_{\pi\left(C_{k+1}^{l}, \widehat{C}_{k+1}^{l}\right)}\left(d_{C_{k+1}^{l}}(\mathbf{x})+d_{\widehat{C}_{k+1}^{l}}(\mathbf{x})\right)^{p-1} d \mathbf{x}\right)^{1 / p}
$$

where $\widehat{C}_{k+1}^{l}=A_{k}\left(C_{k}^{l}\right), d_{C_{k+1}^{l}}$ and $d_{\widehat{C}_{k+1}^{l}}$ are the distance functions of the two curves $C_{k+1}^{l}$ and $\widehat{C}_{k+1}^{l}$.

The temporal intensity smoothness term $E_{i}$ incorporates intensity dynamics defined in Eq.(2) and is chosen to be proportional to the negative log-likelihood of the joint distribution of the intensities, i.e:

$$
E_{i}=-\log \mathbf{p}(\beta)
$$

To minimize the energy function, we developed a coordinate descent algorithm:

- Choose a set of initial curves for $C_{k}^{l}(k=1, \cdots, K ; l=$ $1, \cdots, L)$;

- Assume the curves are known, minimize $E$ with respect to $\beta$;

- Assume the intensity is known, evolve all the curves in the gradient descent direction of $E$ with numerical algorithms of level set methods [5];

- Return to Step 2 until convergence.

\section{EXPERIMENTAL RESULTS}

In this section, we apply our method to reconstruct an image sequence with multiple dynamic objects from sparse Poisson projection data.

The true image sequence was a simulated cardiac sequence generated from the NCAT phantom [11]. It consisted of 16 frames of size $128 \times 128$ covering a cardiac cycle. With multiphase level set methods [12], we used two level set functions at each time to represent four regions in the scene. Each region was assigned a binary label: the heart(01), the liver(10), the thorax region(00), and the background(11). Time varying intensities were also simulated for these four regions as shown in Fig.4. To generate the projection data, we used a SPECT projection matrix generated with the toolbox from [13]. Attenuation, depth dependent blurring, and Poisson noise were simulated. At time $k$, projection data were observed from 6 evenly spaced angles $[11 k, 11 k+30,11 k+60,11 k+90,11 k+120,11 k+150]$ degrees. The signal-to-noise ratio was $20 \mathrm{~dB}$.

The reconstructed image sequence is shown in Fig.2. The reconstructed boundaries are also plotted over the true image sequence in Fig. 3 for comparisons. As we can see, our method correctly reconstructed the boundaries of the left ventricle, the liver and the body contour. We also got very good estimation of the intensities as shown in Fig. 4.

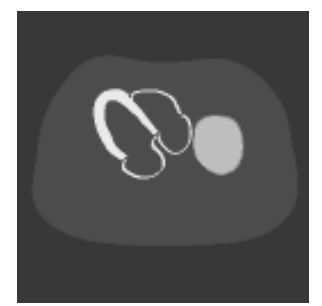

$$
k=1
$$

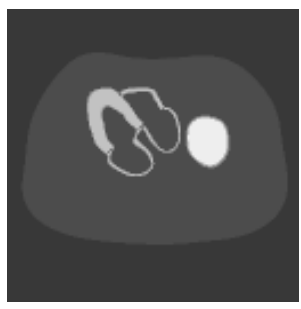

$k=11$

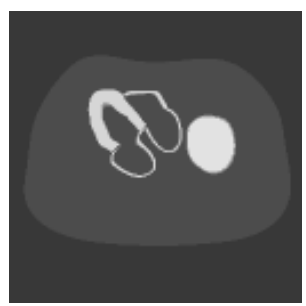

$k=6$

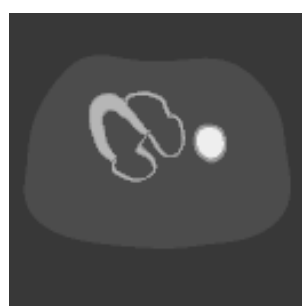

$k=16$
Fig. 1. True image sequence at 4 time instants $(k$ is the time index).

\section{CONCLUSIONS}

In this paper, we presented a variational framework for objectbased dynamic tomography. A general object dynamic model was presented that can capture motion on the object level. This dynamic model was incorporated into the framework with a novel curve distance we proposed. Preliminary experimental results were presented.

\section{REFERENCES}

[1] G.T. Gullberg, R.H. Huesman, S.G. Ross, E.V.R. Di Bella, G.L. Zeng, R.W. Reutter, P.E. Christian, and S.A. Foresti, "Dynamic cardiac single-photon emission computed tomography," in Nuclear Cardiology: State of the Art and Future Directions, B.L. Zaret and G.A. Beller, Eds., pp. 137-187. Mosby, 2nd edition, 1999.

[2] R.E. Carson and K. Lange, "The EM parametric image reconstruction algorithm," Journal of the American Statistical Association, vol. 80, no. 389, pp. 20-22, MAR 1985.

[3] T. Farncombe, A. Celler, C. Bever, D. Noll, J.Maeght, and R. Harrop, "The incorporation of organ uptake into dynamic SPECT(dSPECT) image recon- 


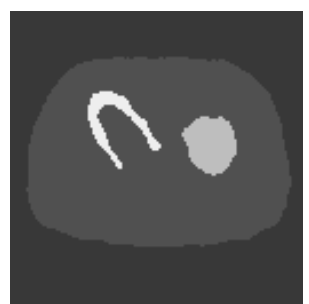

$k=1$

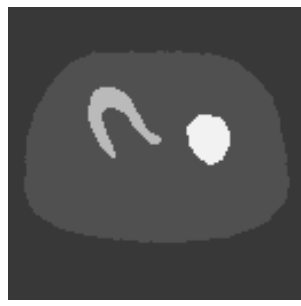

$k=11$

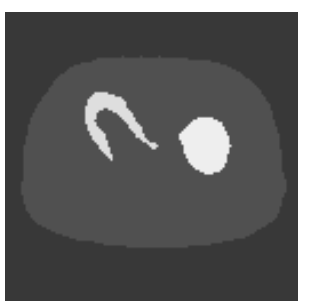

$k=6$

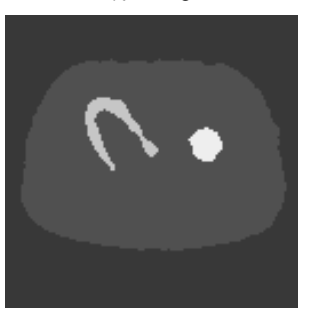

$k=16$
Fig. 2. The reconstructed image sequence at 4 time $\operatorname{instants}(k$ is the time index).

struction," IEEE Trans. on Nuclear Science, vol. 48, no. 1, pp. 3-9, FEB 2001.

[4] D. J. Rossi and A. S. Willsky, "Reconstruction from projections based on detection and estimation of objects-parts I and II: Performance analysis and robustness analysis," IEEE Transactions on Acoustic, Speech, and Signal Processing, vol. ASSP-32, no. 4, pp. 886-906, 1984.

[5] S. Osher and J.A. Sethian, "Fronts propagation with curvature-dependent speed: algorithms based on Hamilton-Jacobi formulations," Journal of computational physics, vol. 79, pp. 12-49, 1988.

[6] A. Litman, D. Lesselier, and F. Santosa, "Reconstruction of a two-dimensional binary obstacle by controlled evolution of a level-set," Inverse Problems, vol. 14, pp. 685-706, 1998.

[7] H. Feng, W.C. Karl, and D.A. Castañòn, "A curve evolution approach to object-based tomographic reconstruction," IEEE Trans. on Image Processing, 2003.

[8] P.C. Chiao, W.L. Rogers, N.H. Clinthorne, J.A. Fessler, and A.O. Hero, "Model-based estimation for dynamic cardic studies using ECT," IEEE Trans. on Medical Imaging, vol. 13, no. 2, pp. 217-226, JUN 1994.

[9] G.S. Cunningham, K.M.Hanson, and X.L.Battle, "Three-dimensional reconstructions from low-count SPECT data using deformable models," Optics Express, vol. 2, no. 6, pp. 227-236, 1998.

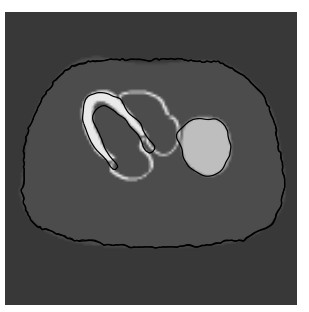

$k=1$

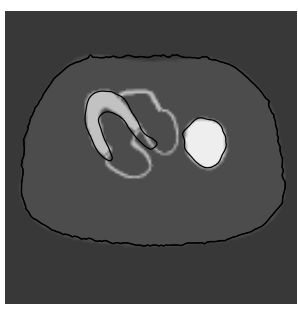

$k=11$

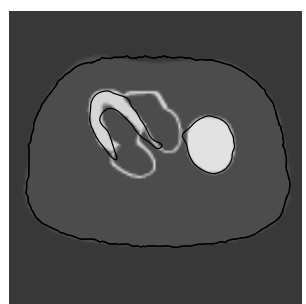

$k=6$

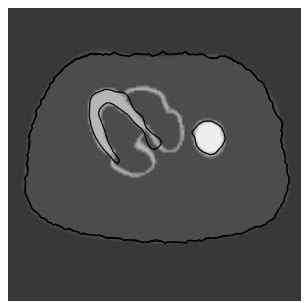

$k=16$
Fig. 3. The reconstructed object boundaries compared with the true image sequence at 4 time instants $(k$ is the time index).

[10] D. Mumford and J. Shah, "Boundary detection by minimizing functionals," in Proceedings of CVPR, San Francisco, June 1985, pp. 22-26.

[11] W.P. Segars, D.S. Lalush, and B.M.W. Tsui, "A realistic spline-based dynamic heart phantom," IEEE Trans. Nucl. Sci., vol. 46, pp. 503-506, 1999.

[12] L.A. Vese and T.F. Chan, "A multiphase level set framework for image segmentation using the mumford and shah model," International Journal of Computer Vision, vol. 50, no. 3, pp. 271-293, 2002.

[13] J.A. Fessler, ASPIRE 3.0 user's guide: a sparse iterative reconstruction library, 2002.

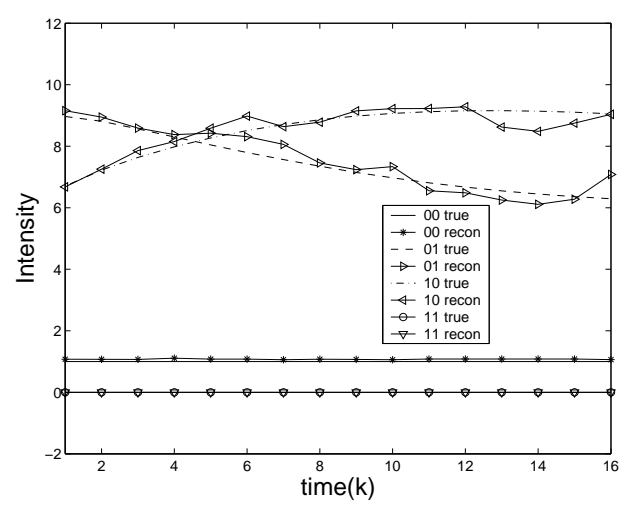

Fig. 4. The reconstructed intensities compared with the true intensities. Each region is identified with a binary label. 